\title{
The Physical Entity of Vector Potential in Electromagnetism
}

\author{
Vladimir A. Leus ${ }^{1}$, Ray T. Smith ${ }^{2} \&$ Simon Maher ${ }^{3}$ \\ ${ }^{1}$ Sobolev Institute of Mathematics, Novosibirsk, Russia \\ ${ }^{2}$ Wirral Metropolitan College, Wirral, UK \\ ${ }^{3}$ Department of Electrical Engineering and Electronics, University of Liverpool, Liverpool, UK \\ Correspondence: Vladimir A. Leus, Sobolev Institute of Mathematics, 630090, Novosibirsk, Russia. E-mail: \\ V.Leus@liverpool.ac.uk, vladalex@liv.ac.uk
}

Received: April 18, 2013 Accepted: June 4, 2013 Online Published: July 10, 2013

doi:10.5539/apr.v5n4p56 URL: http://dx.doi.org/10.5539/apr.v5n4p56

\begin{abstract}
The scalar and vector potentials were introduced into electromagnetic physics in the second half of the nineteenth century. The chief aim was to use them as auxiliary mathematical quantities in order to solve certain practical problems. Nevertheless the discovery of the Aharonov-Bohm effect (1959) in quantum mechanics has suggested that vector potential rather than magnetic field is the causal agent in such an effect. Recent research on the Maxwell-Lodge paradox--induction of voltage in the loop circling a long solenoid carrying alternating current--has confirmed that induction occurs in a region of space effectively free from magnetic field. This again reinforces the idea of vector potential as a physical entity rather than the auxiliary artificial quantity of classical electrodynamics. The present investigation is intended to provide some degree of corroboration of the previous result. The experimental arrangement consists of a 'special' transformer containing movable, single turn coils wound onto rectangular frames. The primary coil is powered from a signal generator providing alternating current over a variable frequency range while the secondary output voltage is connected across a C.R.O./precision voltmeter. Measurements of transformer e.m.f. were carried out at several frequencies in the range $100 \mathrm{~Hz}-20 \mathrm{kHz}$ and with various conditions of shielding around the primary and secondary coils. Certain additional experiments were carried out with a long solenoid and torus solenoid supplied with different core materials. Experimental results for induced e.m.f's are presented and in special cases correlated with the calculated values of mutual inductance. Overall the results tend to confirm the primacy of vector potential over magnetic field as an explanation of the phenomenon.
\end{abstract}

Keywords: electromagnetic induction, transformer e.m.f., vector potential, Maxwell-Lodge paradox, Aharonov-Bohm effect

PACS: 03.50.De Classical electromagnetism, Maxwell equations.

\section{Introduction}

The notion of potentials, both scalar $\varphi$ and vector $\mathbf{A}$, was introduced into electromagnetic physics as auxiliary quantities to facilitate calculation. In particular the potentials allowed Maxwell's system of differential equations of the first order to be transformed into a simpler form so as to solve second order equations, e.g. Poisson'sequation, the wave equation and d'Alambert's equation (Grant \& Phillips, 1998, pp. 30-32, 149-150, $229,433-434)$. Once potentials are obtained, the vectors of magnetic field strength $\mathbf{B}$ and of electric intensity $\mathbf{E}$ may be calculated by simple differentiation. R. A. Mould states: "Prior to the rise of gange theory and the discovery of the Aharonov-Bohm effect, physicists tended to regard these potentials as fictitious. Much was made of the fact that electromagnetic measurement always involve forces that manifest themselves in the Lorentz force equation and, hence, that the $\mathbf{B}$ and $\mathbf{E}$ fields appearing in that equation are the "real "fields. Because the potentials cannot be uniquely determined from $\mathbf{B}$ and $\mathbf{E}$, they were said not to share in this reality" (Mould, $\mathrm{p}$. 167). However after discovery of the Aharonov-Bohm effect (Aharonov \& Bohm, 1959), quantum mechanics tended to regard vector potential rather than the magnetic field as the casual agent in any explanation of this effect. The electromagnetic potentials that are components of the four-vector potential $\mathbb{A}=(\mathbf{A}, i \varphi / c)$ now become the fundamental variables of the theory of quantum mechanics. Classical electromagnetism proves to be more conservative regarding the acceptance of this point of view, but recent investigations have contributed to the scientific revival of the problem which has led to a renewed interest in this topic. 
The transformer is a simple device to set up. It could hardly be less sophisticated, without the need of moving parts, permanent magnets, sliding contacts, vacuum tubes etc. Despite the simplicity of such a device, the theory of its operation has raised fundamental questions regarding the very foundation of electromagnetism. This was first pointed out and experimentally researched more than a century ago by Oliver Lodge (Lodge, 1889). Rousseaux et al. (2008) emphasise that the Maxwell-Lodge effect still presents a fundamental problem. A very long solenoid is circled in its central plane by a conducting loop (Figure 1). When a sinusoidal current is supplied to the solenoid, there is a corresponding voltage induced in the loop, despite the fact that no sensible magnetic field exists in the vicinity of the loop. So the question is posed: "How do the charge carriers in the external coil know that the current is varying in the solenoid?" (Rousseaux et al., 2008, p. 251).

It is of interest, from an historical point of view, that Sir Oliver Lodge over 120 years ago had pondered a similar question. In 1889, at University of Liverpool, Lodge carried out an experiment using a torus solenoid wound onto a ring shaped iron core. In his own words: "So I led a short wire round the outside of the ring and brought its ends into the box where the needle was, one on each side of the needle. A distinct and proper deflexion was now observed. A wire was then taken 10 times around the ring, and connected to a common quadrant electrometer. The deflexion was easy to see. It could also be just seen with only one turn of wire" (Lodge, 1889, pp. 476-77). The phenomenon associated with induction of e.m.f. in a magnetic field free region might then be appropriately termed the 'Maxwell-Lodge Effect'. Furthermore, in his concluding remarks, Lodge makes a reference to what effect some form of electromagnetic shielding might have between two circuits: "I would not wish the Society to suppose that I regard the research as finished and complete. I should like to clear up distinctly the effect of screens" (Lodge, 1889, p. 479). So we might regard the present investigation as a continuation of Lodge's work, using shielded and unshielded rectangular circuits in addition to work with solenoidal and toroidal coils.

It should be noted that Lodge was faced with considerable experimental difficulties by contrast with today. For example he could only detect (stray) magnetic fields by the use of iron filings; alternating voltage was simulated by including a reversing key in a direct current circuit; induced voltage was detected using a quadrant electrometer plus lamp and scale. Such an instrument was very sensitive to extraneous vibrations: "There remained a few irregular disturbances, some of which could be traced to convection-currents, others to the ordinary movements about a building, and others again to the passage of London and North-Western trains in their tunnel, some 150 yards away and 60 feet down, in the sandstone rock" (Lodge, 1889, p. 475). Experimentation was certainly a much more painstaking ordeal in those days.

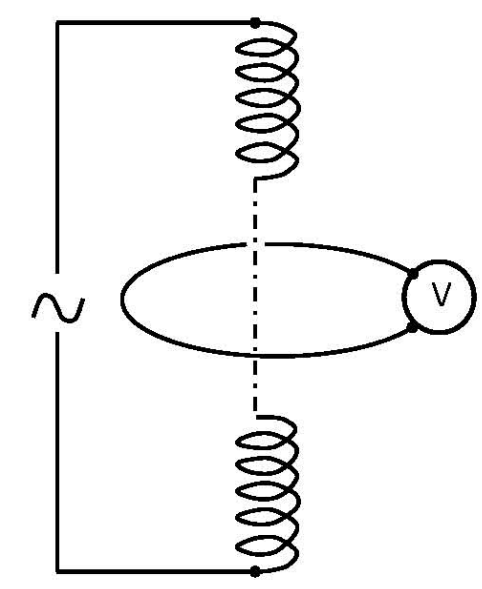

Figure 1. Circuits for the Maxwell-Lodge effect representation

Rousseaux et al. (2008) used for their experimentation a diversity of Lodge's apparatus with voltmeter instead of a movable needle (Figure 1). They attempt to answer above question in the following way. Each vector field $\mathbf{A}$ may be decomposed into three additive parts: $\mathbf{A}=\mathbf{A}_{\|}+\mathbf{A}_{\perp}+\mathbf{A}_{h}$, where the third one (so called harmonic part) meets the conditions $\operatorname{div} \mathbf{A}_{h}=0, \operatorname{curl} \mathbf{A}_{h}=0$. If $\mathbf{A}$ is vector potential, then its harmonic part is responsible for the presence in the loop of an electric field $\mathbf{E}=-\partial_{t} \mathbf{A}_{h}$. Since the harmonic part is a gradient, such an inference contradicts the conventionally adopted "gauge condition principle", which states that it may be 
removed by subtracting the gradient of an appropriate scalar gauge function. The authors of (Rousseaux et al., 2008) regard the "gauge principle" as incorrect and refer to the Aharonov-Bohm effect, which is explained in terms of the harmonic part $\mathbf{A}_{h}$ of the vector potential. In (Rousseaux et al., 2008) an "experimentum crucis" corroborating this point is presented, and the authors propose to replace the generally adopted term "gauge condition" by "gauge constraint". The significant feature of the experimental results presented in (Rousseaux et al) is that the exterior of the solenoid coil provides a region in which the magnetic field is close to zero whereas the vector potential assumes a definite value. In the present paper a different geometrical form of the same experiment is investigated which tends to validate the conclusion of (Rousseaux et al., 2008) but allows a further insight into this fundamental problem. Here the apparatus used for showing the Maxwell-Lodge effect includes a special transformer having two identical loops for both the primary and secondary circuits. This configuration allows us to operate under two different sets of experimental conditions, (1) with free magnetic field access (i.e. using 'unshielded' loops) and (2) without magnetic field access (i.e. using 'shielded' loops). As regards multi-turn coils, we used a long solenoid (similar to one in Rousseaux et al., 2008); in addition we carried out experiments with two types of fully wound torus solenoid. It is appropriate to mention that we worked in the quasi-static limit where retardation effects are negligible.

\section{Experimental Arrangement}

The basic arrangement consists of single rectangular loops of stranded wire taped onto rectangular wooden frames of the same dimensions, $1.20 \times 0.60 \mathrm{~m}$. One loop (the primary) is powered by a signal generator and power amplifier capable of providing an alternating current upto 10 A peak ( 7.07 A r.m.s) and up to $20 \mathrm{kHz}$ frequency. The induced voltage across the second loop (secondary) is measured on a dual beam oscilloscope (Hameg) in conjunction with a precision a.c. digital voltmeter (Hameg).

The geometrical arrangement of the two loops can be varied in two ways. In Figure 2(a) the loops are located one above the other. Here the vertical separation of the loops $(d)$ can be varied using small wooden spacers between frames. In Figure 2(b) the lateral separation of the loops can be varied likewise. Furthermore, one or both loops can be shielded using soft iron or thin mu metal tubing. Further experiments were carried out with a long solenoid using four different types of core (wood, iron, aluminium and copper) and for two torus solenoids with different types of core (plastic and iron). The experimental results for all the above arrangements are presented in section 4.

\section{Theoretical Estimations}

\subsection{Vertical Separation of Rectangular Loops}

The experimental values of induced voltage can, in certain cases, be compared with calculated values based on particular geometrical configurations. Neumann gives a general expression for calculating mutual inductance of circuits (1) and (2) as

$$
M_{12}=\frac{1}{4 \pi \varepsilon_{0} c^{2}} \oint \oint \frac{d s_{1} \cdot d s_{2}}{r_{12}},
$$

where $r_{12}$ is the separation of line elements $d s_{1}$ and $d s_{2}$ in the circuits (1) and (2). So it follows that e.m.f. $\left(e_{2}\right)$ induced in circuit $(2)$ by a varying current $(I)$ in circuit (1) is $e_{2}=M_{12} d I_{1} / d t$. Now it is possible to calculate $M$ for specific geometries. In particular Gray $(1967$, p. 497) gives a formula for calculating the mutual inductance of co-axial equal, parallel rectangles composed of thin conductors, with sides $a$ and $b$, distance $d$ apart (Figure 2a). The value of $M$, in $\mu H$ when $a, b$ and $d$ are measured in $c m$, is given by

$$
\begin{array}{r}
M=4\left[a \ln \left\{\frac{a+\left(a^{2}+d^{2}\right)^{1 / 2}}{a+\left(a^{2}+b^{2}+d^{2}\right)^{1 / 2}} \frac{\left(b^{2}+a^{2}\right)^{1 / 2}}{d}\right\}+b \ln \left\{\frac{b+\left(b^{2}+d^{2}\right)^{1 / 2}}{b+\left(a^{2}+b^{2}+d^{2}\right)^{1 / 2}} \frac{\left(a+d^{2}\right)^{1 / 2}}{d}\right\}\right. \\
\left.+8 \ln \left\{\left(a^{2}+b^{2}+d^{2}\right)^{1 / 2}-\left(a^{2}+d^{2}\right)^{1 / 2}-\left(b^{2}+d^{2}\right)^{1 / 2}+d\right\}\right] \times 10^{-3}(\mu H)
\end{array}
$$

Finally, if $I_{1}$ represents a sinusoidal current $I_{1}=I_{0} \sin (2 \pi f t)$, then simple differentiation gives the peak value of the induced e.m.f. $\left(e_{2}\right)_{0}$ as 


$$
\left(e_{2}\right)_{0}=2 \pi f I_{0} M_{12}
$$

So by computing the values of $M$ for a range of $d$ values the induced voltage can be calculated for an alternating current of given magnitude and frequency.

\subsection{Horizontal Separation of Rectangular Loops}

In the case of rectangular loops in the same plane, application of Neumann's formula does not lead to simple analytical expressions for Mutual Inductance. Approximate values for induced e.m.f. in these cases can be estimated from an application of Faraday's Flux linking rule. The induced e.m.f. for non-overlapping, coplanar rectangular coils can then be approximated by calculating the net rate of change of flux through the secondary coil from an alternating current in the primary. An initial upper estimate is obtained by simply treating the primary coil as an infinite straight wire. The peak e.m.f. in the secondary is then given as:

$$
e_{\text {peak }}=-\mu_{0} I_{0} \text { af } \ln \left(\frac{s}{s-b}\right) .
$$

When the longer sides of the loop are treated as infinite wires and the (oblique) contributions from the shorter sides are neglected, the peak e.m.f. in the secondary is given as:

$$
e_{\text {peak }}=\mu_{0} I_{0} \text { af }\left[\ln \left(\frac{b+s}{s}\right)-\ln \left(\frac{s}{s-b}\right)\right],
$$

where $\mu_{0}$ is permeability of free space, $I_{0}$ is peak current, $a$ is loop length, $f$ is frequency, $b$ is loop width, and $s$ is the loops separation distance (Figure 2b).

\section{The Experimental Data}

The basic quantitative measurement has been carried out with the rectangular wooden frames fitted with $10 \mathrm{~A}$ rated multi-stranded copper wire.

The first experimental measurement was done for different vertical positions (Figure 2a) and for different horizontal positions (Figure 2b) of the secondary frame relative to the primary. The distance

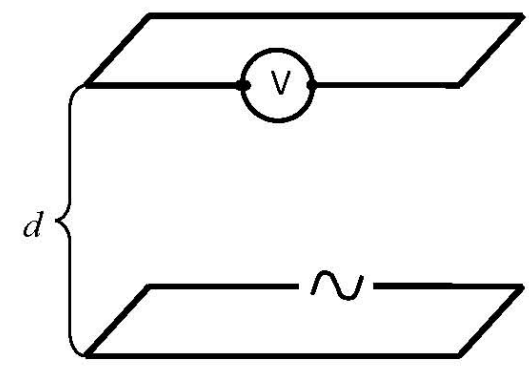

(a)

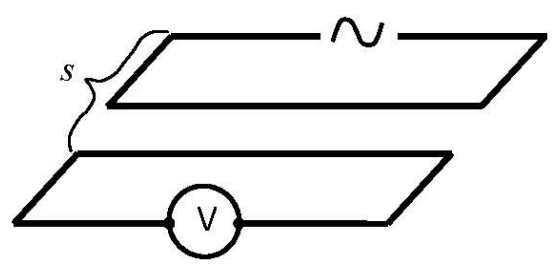

(b)

Figure 2. Scheme of the first experimental measurement

$d$ determines a position of the movable frame and e.m.f. is measured with a milli-voltmeter. The working frequency and current were $1 \mathrm{kHz}$ and 5 Amps (r.m.s.) respectively. The results of measurement versus theoretical calculation are shown in Figure 3 for the case of the vertical shifting, and in Figures 4 and 5 for the case of horizontal shifting. 


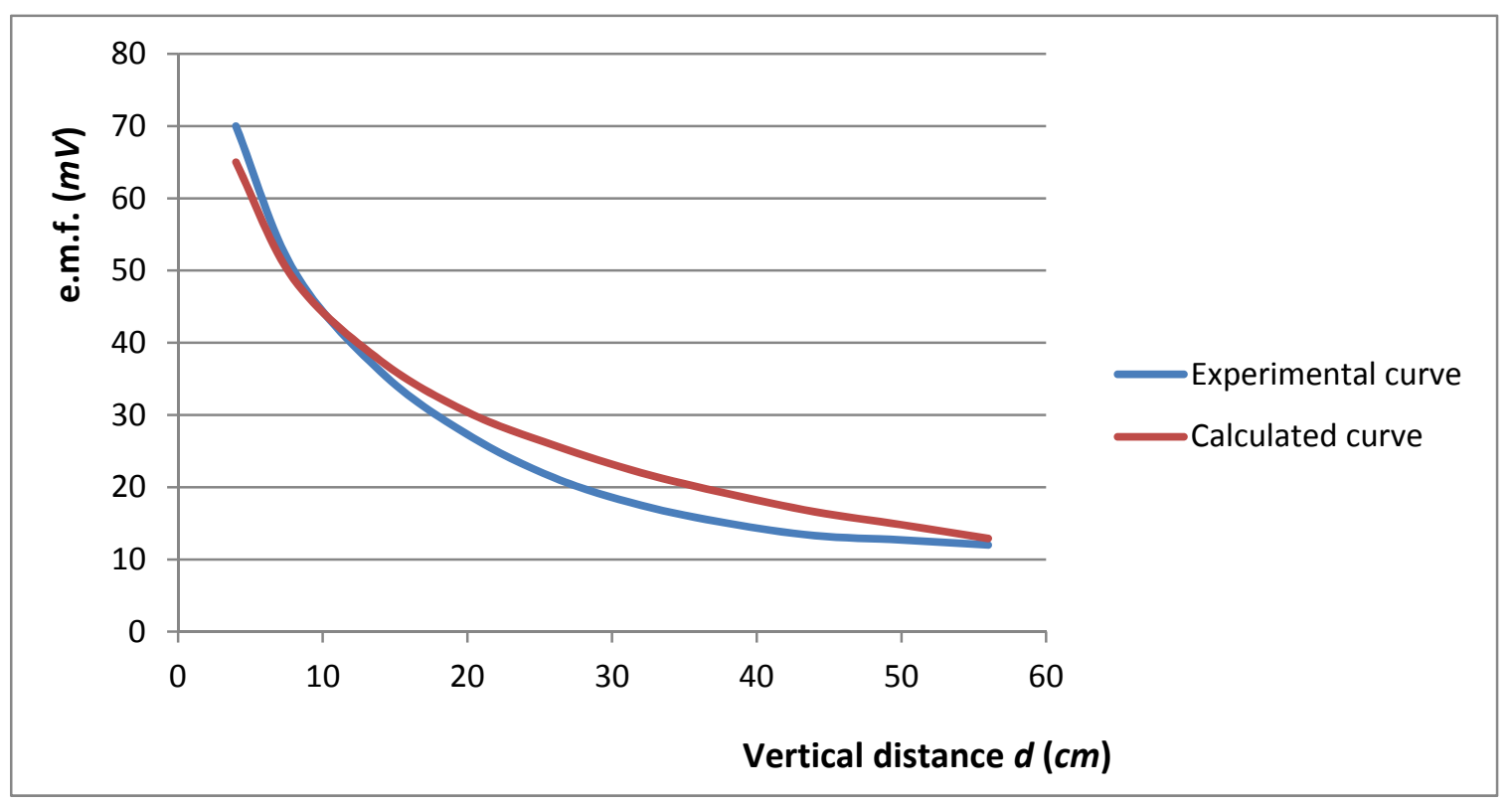

Figure 3. Experimental and calculated voltage depending on the vertical shift

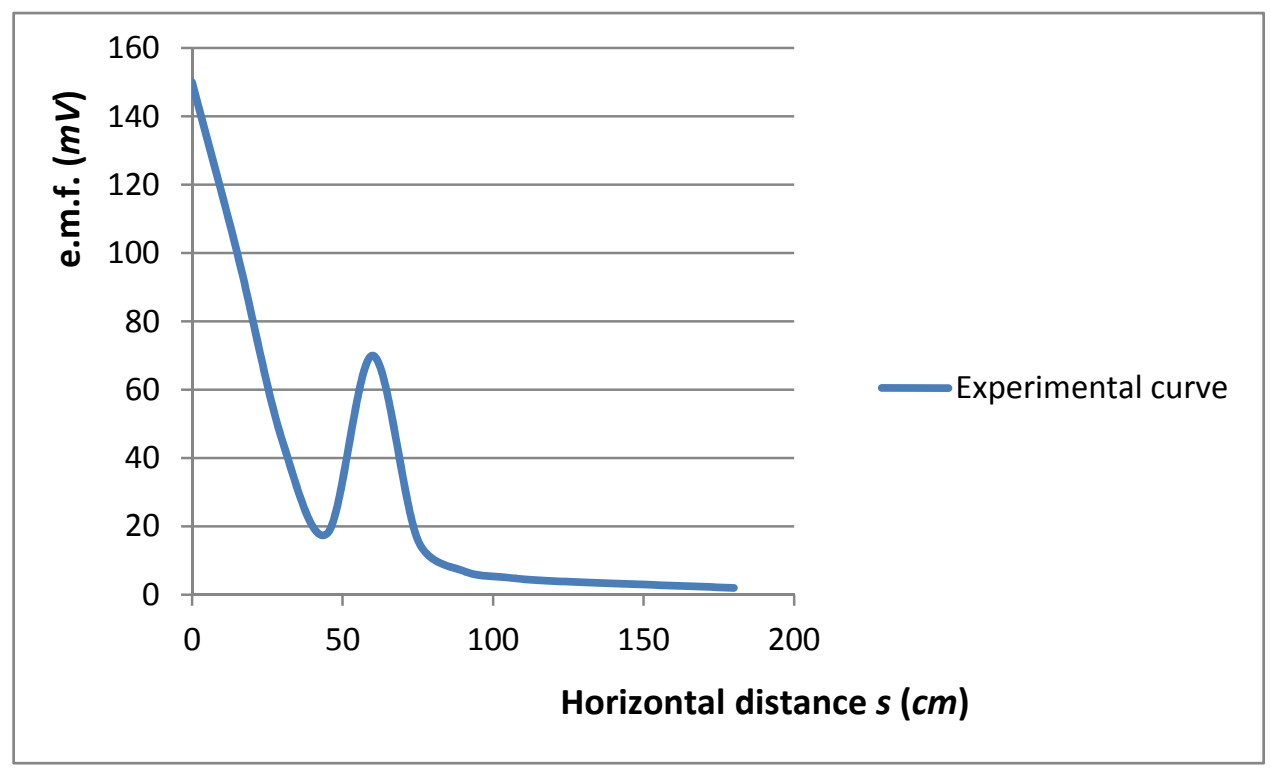

Figure 4. Experimental voltage depending on the horizontal shift 


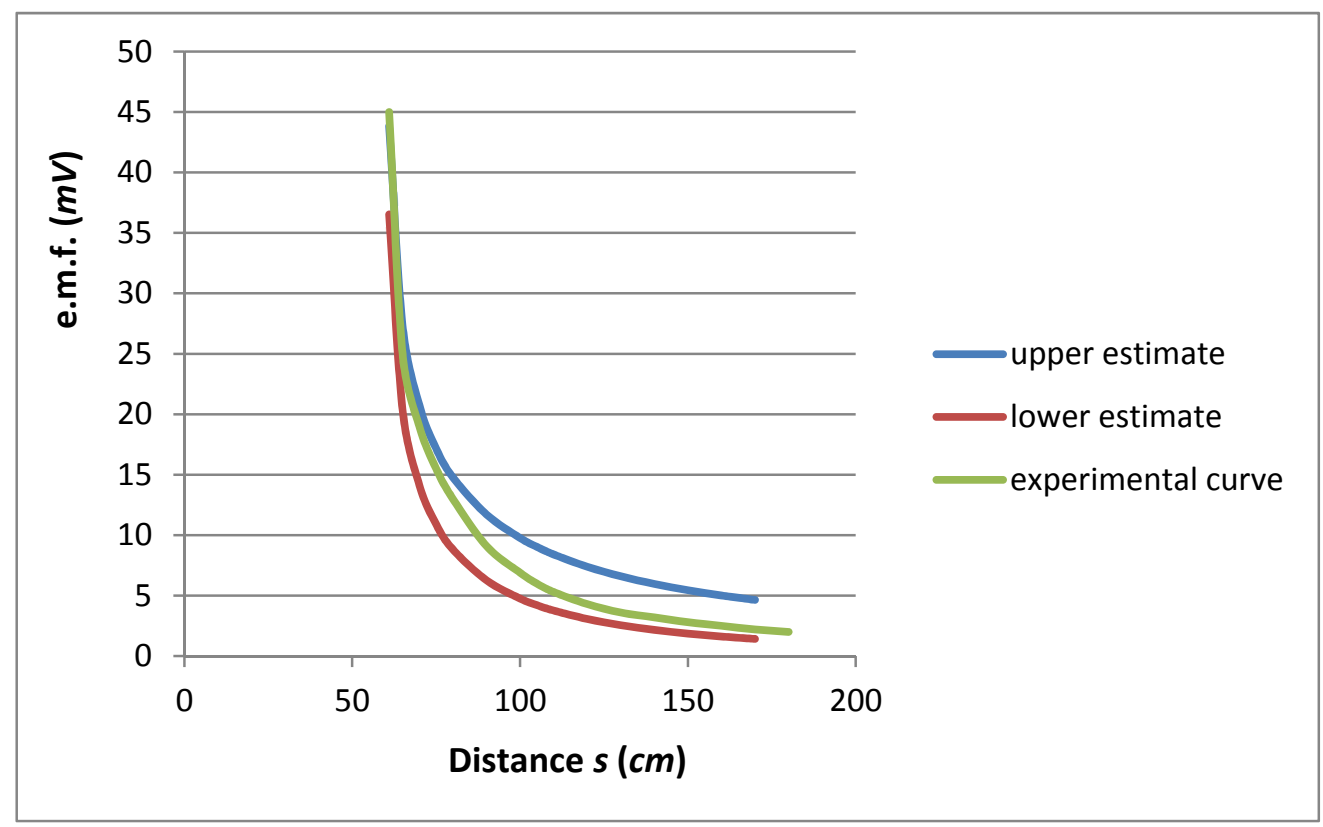

Figure 5. Calculated and measured e.m.f. depending on the horizontal shift

The second experimental measurement was made with one long side of the secondary rectangular frame screened with three layers of iron tubing (Figure 6a). This is depicted in Figure 6b where the second half of the square tube is taken off. All layers are insulated from each other. No significant shielding effect was indicated. The same result was obtained with the screen earthed and after the screen was swapped to the primary frame. For comparison we substituted this multi-layer screen for an aluminium tube of $7 \mathrm{~mm}$ wall thickness. There was no appreciable change in the result regardless of whether the tube was earthed or not.

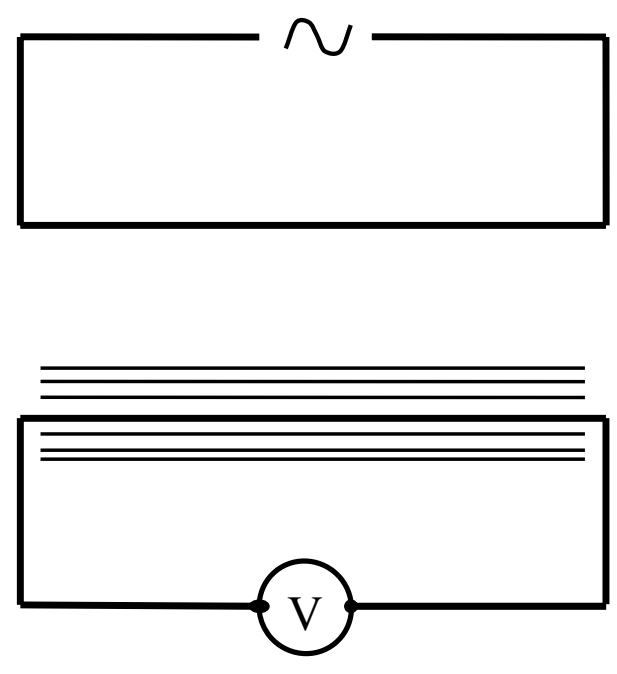

(a)

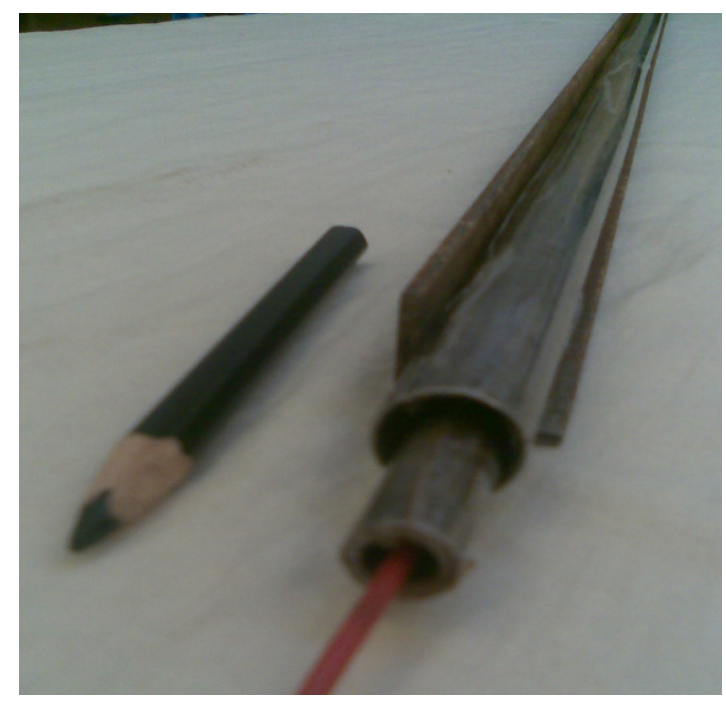

(b)

Figure 6. Three layer shielding

The third experimental measurement was performed for the full screening of one frame enclosed by a rectangular-bent iron tube of 1" outer diameter and wall thickness two millimetres (Figure 7). Although the external magnetic field from a direct current flowing in the wire was reduced several times, no appreciable shielding effect was observed when the transformer worked with alternating current. The same result was 
observed whether the full screening was around the primary or secondary and regardless of the screen being earthed or not.

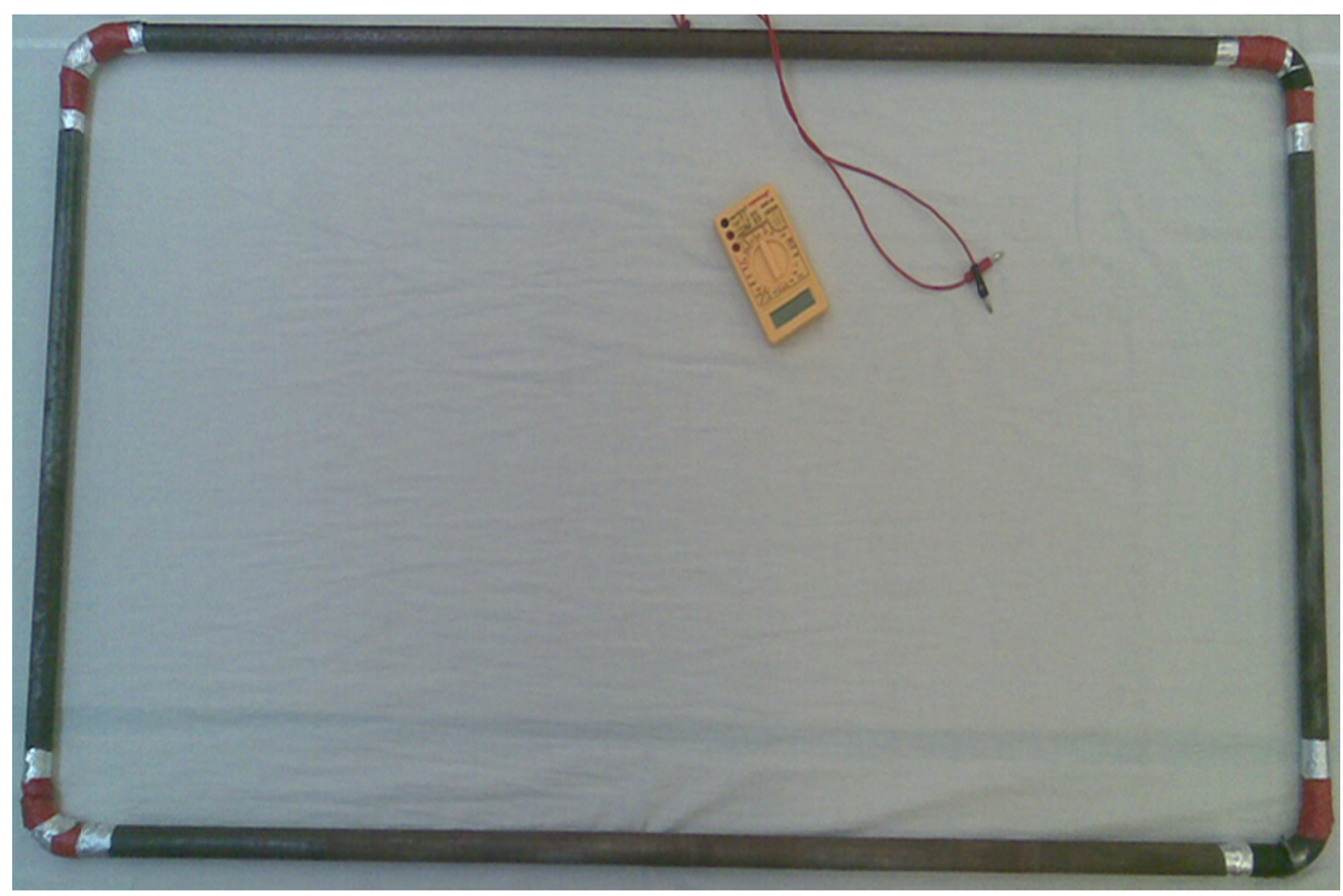

Figure 7. Full screened rectangular loop

The fourth experimental measurement was performed for a long solenoid (length, L) with length:diameter ratio equal to 25 , wound with copper wire of $1 \mathrm{~mm}$ diameter (Figure 8). A loop of radius $R$ played the role of the secondary circuit. In the case of a long solenoid the influence of the diameter is not appreciable when $R<L$, and the induced voltage gradually decreased when $R>L$. Such behaviour is understandable because all the magnetic field is confined inside the long solenoid, but the leakage field is significant for the shorter type. Four different materials were used for the core: wood, iron, aluminium, and copper (Figure 8a). For all the types of core the initial current was set at 5 Amps (r.m.s.). Wood and copper gave the same result as when no core is present (air). In the case of aluminium the induced voltage was reduced by several percent. When an iron core is introduced inside the solenoid, the current reduces abruptly to around 2 Amps (r.m.s.), and the voltage increased several times in magnitude. In both cases - aluminium and iron-appreciable heating was noted. In fact, after two minutes the iron rod became impossible to touch with the naked fingers, whereas the aluminium rod itself felt rather tepid.

One more version of the experiment was carried out with the iron core closed into a rectangle (Figure 8b). No essential differences were noted, although in this case only a small part of the magnetic field remains in the free space. Finally, a fifth experiment was carried out for a fully wound torus solenoid. 

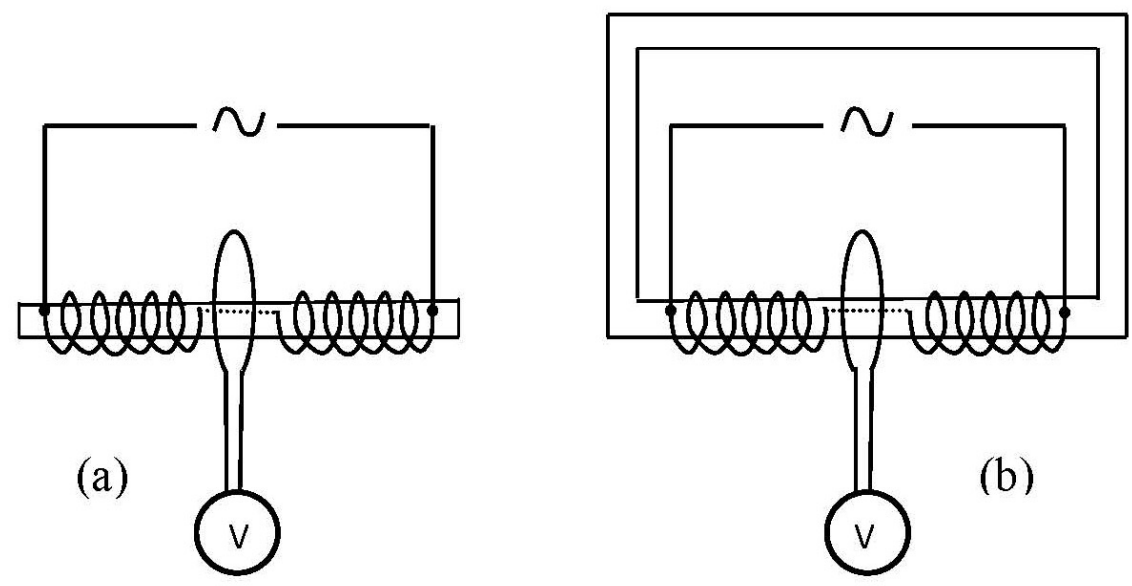

Figure 8. Long solenoid with core

The fifth experimental measurement was carried out using torus solenoids wound with copper wire $0.5 \mathrm{~mm}$ in diameter (Figure 9). Two types of core were used: plastic (bigger torus) and iron. At $10 \mathrm{kHz}$ frequency and 1 Amp current intensity, the e.m.f. in secondary loop for the first solenoid was $80 \mathrm{mV}$ and for the second solenoid- $600 \mathrm{mV}$ (peak-to-peak). In this absolutely closed condition the main question is especially remarkable: "How does the external loop know about the type of core confined inside the full-wound torus solenoid?"

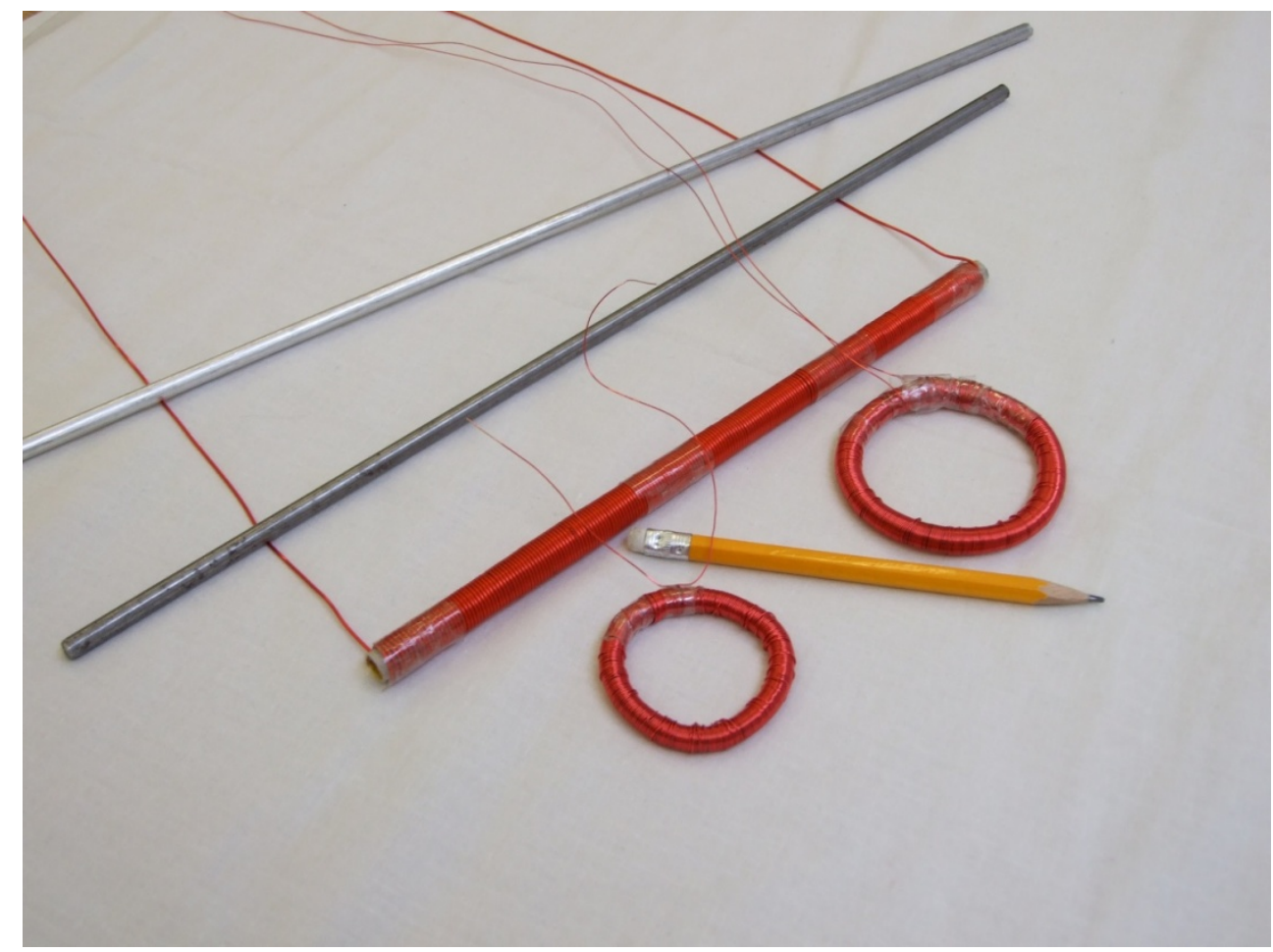

Figure 9. Long solenoid with different cores and two torus solenoids

\section{The Role of Vector Potential in Electromagnetic Transmission}

The evidence from the long solenoid (Rousseaux et al., 2008) and torus coil (Tonomura A.) carrying alternating current, lends support to the view that an electromagnetic influence can travel through a space, free of a magnetic field. This effect is verified by the operation of transformers on a worldwide scale. What inference can be drawn from the experiments described relating to the shielding of circuits carrying alternating current? Our 
experimental findings seem compatible with the results presented in (Rousseaux et al., 2008) given that a constant magnetic field plays no role in the mechanism of electromagnetic transmission. Rousseaux et al propose to consider the harmonic part of vector potential to be accountable for propagation, whereas our suggestion is to regard the vector potential $\mathbb{A}$ in total as playing a part in the process.

Consider a very long straight wire with a direct electrons' current $\boldsymbol{j}=(0,0, j)$ flowing along $z$-axis (Figure 10). Let the vector potential be defined as a vector directed along the wire:

$\mathbf{A}=\left(0,0, j \ln \sqrt{x^{2}+y^{2}}\right)$. Then the magnetic field strength is

$$
\mathbf{H}=\operatorname{curl} \mathbf{A}=\left(\frac{\partial}{\partial y} j \ln \sqrt{x^{2}+y^{2}},-\frac{\partial}{\partial x} j \ln \sqrt{x^{2}+y^{2}}, 0\right)=\left(\frac{j y}{x^{2}+y^{2}}, \frac{-j x}{x^{2}+y^{2}}, 0\right) .
$$

The calculation of the partial derivatives gives:

$$
\begin{aligned}
\frac{\partial}{\partial x} H_{x} & =-j \frac{2 x y}{\left(x^{2}+y^{2}\right)^{2}}, \quad \frac{\partial^{2}}{\partial x^{2}} H_{x}=2 j y \frac{3 x^{2}-y^{2}}{\left(x^{2}+y^{2}\right)^{3}} \\
\frac{\partial}{\partial y} H_{x} & =-j \frac{x^{2}-y^{2}}{\left(x^{2}+y^{2}\right)^{2}}, \quad \frac{\partial^{2}}{\partial y^{2}} H_{x}=-2 j y \frac{3 x^{2}-y^{2}}{\left(x^{2}+y^{2}\right)^{3}} \\
\frac{\partial}{\partial x} H_{y} & =-j \frac{x^{2}-y^{2}}{\left(x^{2}+y^{2}\right)^{2}}, \quad \frac{\partial^{2}}{\partial x^{2}} H_{y}=2 j y \frac{3 y^{2}-x^{2}}{\left(x^{2}+y^{2}\right)^{3}} \\
\frac{\partial}{\partial y} H_{y} & =j \frac{2 x y}{\left(x^{2}+y^{2}\right)^{2}}, \quad \frac{\partial^{2}}{\partial y^{2}} H_{y}=-2 j x \frac{3 y^{2}-x^{2}}{\left(x^{2}+y^{2}\right)^{3}} .
\end{aligned}
$$

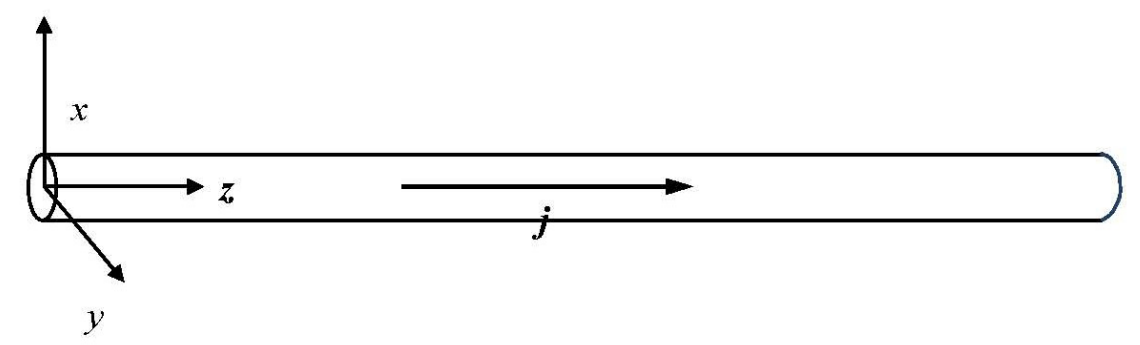

Figure 10. Direct Current in a straight wire

If now the wire carries alternating current $\boldsymbol{j}=(0,0, J \sin \omega t)$ the vector potential will be $\mathbf{A}=(0,0$, $J \sin \omega t \ln \sqrt{x^{2}+y^{2}}$, and the magnetic field strength

$$
\mathbf{H}=J \sin \omega t\left(\frac{y}{x^{2}+y^{2}}, \frac{-x}{x^{2}+y^{2}}, 0\right),
$$

and its partial derivatives over time are

$$
\frac{\partial}{\partial t} \mathbf{H}=J \omega \cos \omega t\left(\frac{y}{x^{2}+y^{2}}, \frac{-x}{x^{2}+y^{2}}, 0\right), \quad \frac{\partial^{2}}{\partial t^{2}} \mathbf{H}=-J \omega^{2} \sin \omega t\left(\frac{y}{x^{2}+y^{2}}, \frac{-x}{x^{2}+y^{2}}, 0\right) .
$$

The Laplasian operator $\nabla^{2}$ acting on vector $\mathbf{H}$ gives $\frac{\partial^{2} \mathbf{H}}{\partial x^{2}}+\frac{\partial^{2} \mathbf{H}}{\partial y^{2}}+\frac{\partial^{2} \mathbf{H}}{\partial z^{2}}$ and it may be easily calculated using formulae (1): $\nabla^{2} \mathbf{H}=\left(\nabla^{2} H_{x}, \nabla^{2} H_{y}, \nabla^{2} H_{z}\right)=(0,0,0)=\mathbf{0}$. Hence, the wave operator acting on $\mathbf{H}$ gives the following expression (where $\mathrm{c}$ is the speed of light): 


$$
\nabla^{2} \mathbf{H}-\frac{1}{c^{2}} \frac{\partial^{2}}{\partial t^{2}} \mathbf{H}=\frac{J \omega^{2} \sin \omega t}{\mathrm{c}^{2}}\left(\frac{y}{x^{2}+y^{2}}, \frac{-x}{x^{2}+y^{2}}, 0\right) \neq \mathbf{0} .
$$

It is evident that a variation of magneto-static field does not propagate as a wave. Whether static magnetism is present or not on the scene - that does not matter. However it is highly plausible that electro-dynamical flow of energy is related to the time variation of vector potential.

In his paper "On an electrostatic field produced by varying Magnetic induction" dated from 1889 Oliver Lodge poses the question relating to the possible existence of different types of induction: "In common no doubt with many others, I have long wished to find some connection between static electricity and magnetism. My early notions in this direction were such ideas as the following: (1) To spin a long bar-magnet on its axis, suspending in its field a sort of quadrant-electrometer needle charged oppositely at either end, and to look at deflection. The iron core of an electromagnet was supposed to serve. ... (3) To use a varying magnet instead of a moving one;... for instance, to suspend a charged gold leaf between the edges of the pole-pieces of an electromagnet, and watch it through a microscope when the exiting current is started or stopped" (Lodge, 1889, p. 469). He proposed two scientific hypotheses over the behaviour of a charge moving in a magnetic field. One of them concerned the problem of relativity: "... think of a charged gold leaf hanging between the poles of magnet. If the gold leaf moves it constitutes a current and therefore at once feels a force urging the current (i.e. the line of motion) across the lines of force in the orthodox way. Instead of supposing the gold leaf to move, let the magnet move. The same thing will happen, because the relative motion is the same as before" (Lodge, 1889, p. 470). More than a century after Lodge this topic remained a subject for controversy between eminent physicists with some Nobel laureates included (Leus \& Zatolokin, 2006). Hitherto some highly sophisticated experiments have been suggested and tried (Zajev \& Dokuchajev, 1964; Bogach, 1996; Kelly, 2004; Rajaraman, 2008; Leus \& Taylor, 2011; Taylor \& Leus, 2012), which confirmed the correctness of Lodge's view.

The second hypothesis dealt with electromagnetic induction: "Or instead of moving the magnet, let its strength be varied; lines of force pass by the charged body, unless it is symmetrically situated, in this case as in the other; and hence the effect may naturally be expected to be the same in either case" (Lodge, 1889, p. 470). In this respect of reciprocal behaviour among charge and field Lodge is incorrect because he fails to distinguish between dynamical and kinematical origins of induction (Leus \& Taylor, 2012).

If a permanent magnet moves with a constant velocity through the laboratory then, although the vector potential varies at each point, no electromagnetic disturbance propagates through space with the speed of light. It is because of the charge's acceleration that "gauge fields", $\mathbf{A}$ and $\varphi$, are simultaneously varying in parallel with the electromagnetic field. In the Gaussian system of units we have

$$
\mathbf{E}(t)=-\left(\operatorname{grad} \varphi+\frac{1}{c} \frac{\partial}{\partial t} \mathbf{A}\right), \quad \mathbf{H}(t)=\operatorname{curl} \mathbf{A} .
$$

All these entities are inseparably linked and in total they make up integral parts of a physical unity -- the propagating electromagnetic disturbance. If on the path of such a propagating disturbance an electric charge is encountered then it experiences the action of a force, which we call electromagnetic induction. From an historical point of view it should be emphasised that vector potential was introduced by Maxwell to describe the electro-tonic state of the surrounding medium (aether) based on a continuum state of matter. Post Maxwell we have current associated with free electron drift velocity $\boldsymbol{v}$ and so $d I / d t$ in the induced e.m.f. formula relates directly to electron acceleration.

In the literature from the times of Poynting there has existed prolonged confusion regarding the subject of dynamic behaviour of magnetic field lines of force. For example, to quote Poynting (1885): "I shall suppose, then, that alteration in the quantity of magnetic induction through a closed curve is always produced by motion of induction tubes inwards or outwards through the bounding curve". The diagram in Figure 11 illustrates two principally different mechanism of magnetic transmission. The illustration is split into two regions separated with a common time axis depicting alternating current. The upper shows the "kinematical" point of view, when the magnetic lines of force really move through space. Here we propose an alternative-the very "dynamical" mechanism illustrated in the lower region of Figure 11.

When an electromagnetic disturbance propagates through space, the lines of force do not move anywhere at all. Instead, if at any position the field $\mathbf{H}(t)$ is reducing (increasing), the intensity of lines of force in the local 'induction tube' is decreasing (increasing) and so too the field $\mathbf{E}(t)$-the lower drawing on the Figure 11. 

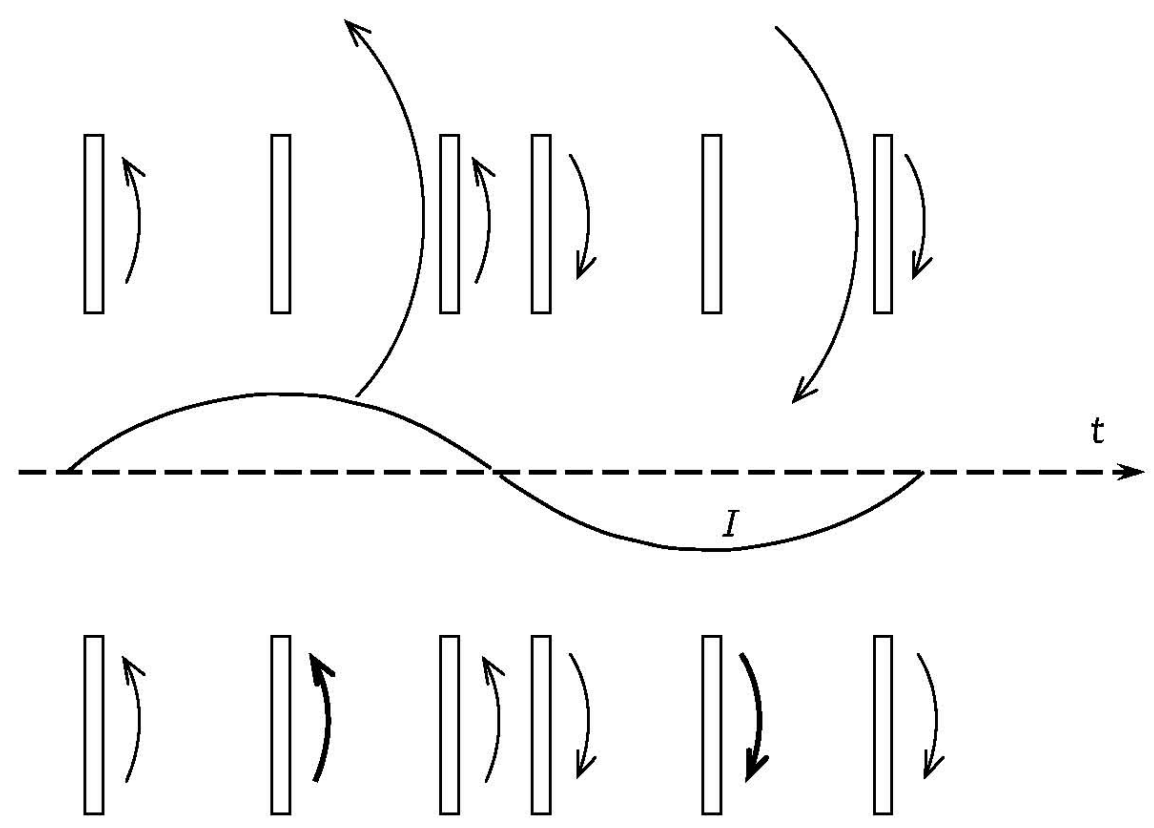

Figure 11. Alternating current in an electromagnet produces variable magnetic field

Thus the acceleration of a charge, which is associated with the creating and propagation of an electromagnetic disturbance, seems inseparably linked with the "trinity" of vectors $(\mathbb{A}, \mathbf{E}, \mathbf{H})$. However, it would be as well to recall that the expression 'induction tube' used by Poynting is little more than a visual aid helping us to comprehend the physical reality. We would go along with the conclusion expressed in (Rousseaux et al., 2008) that "In the case of the perfect solenoid or the torus, the tubes present in the interior of the solenoid do not cut the external coil. For a finite solenoid, of course, the tubes close themselves in the exterior but a current variation in the solenoid does not imply their displacement or the cutting of the coil." Let us add to this, the kinematical induction appears when a permanent magnet is moving by and its lines of force indeed "cut" a conducting wire (Leus \& Taylor, 2012).

\section{Conclusion}

By adopting vector potential as a physical entity it is argued that we have in essence reverted back to Maxwell's views from over 150 years ago. He regarded vector potential as the measure of Faraday's 'electro-tonic state', and therefore as a propagable physical entity. Around 1900 Emil Wiechert, an earlier writer on electrodynamics had commented: "For Maxwell A (vector potential) was not a mere mathematical auxiliary quantity as it is for us but a function of state of especial significance" (O'Rahilly, 1965, p. 184-5). With the advent of Relativity and Quantum mechanics the notion of aether gradually became relegated to a non physical role. In his Lectures on Physics R. Feynman barely mentions the aether of Lodge, Maxwell and Lorentz. He does, however, provide a post Aharonov-Bohm view of electrodynamics when he writes: “...the vector potential appears to give the most direct description of the physics. This becomes more apparent the more deeply we go into quantum theory. In the general theory of quantum electrodynamics, one takes the vector and scalar potentials as the fundamental quantities in a set of equations that replace the Maxwell equations: $\mathbf{E}$ and $\mathbf{B}$ are slowly disappearing from the modern expression of physical laws; they are being replaced by $\mathbf{A}$ and $\varphi$ " (Feynman et al, 1989, chapter 15, section 5).

The experiments presented in this paper are intended to highlight one of the fundamental questions at the heart of electrodynamics, namely: what is the carrier of electromagnetic actions? In general the results obtained allow us to promote the following hypothesis: the four-vector potential $\mathbb{A}=(\mathbf{A}, i \varphi / c)$ is nothing else but a quantitative characteristic of the aether lost in the last century. Its numerical value depends on the "point of view" - a chosen inertial frame of reference, and is given by Lorentz transform equations:

$$
A_{x^{\prime}}=\gamma\left(A_{x}-\beta \varphi / c\right), \quad A_{y^{\prime}}=A_{y}, \quad A_{z^{\prime}}=A_{z}, \varphi^{\prime} / c=\gamma\left(\varphi / c-\beta A_{x}\right)
$$

for a velocity $\boldsymbol{v}=(v, 0,0)$, where $c$ is light speed, $\beta=v / c, \gamma=\left(1-\beta^{2}\right)^{-1 / 2}$ (Mould, 1995, p. 71). 
Following on from Oliver Lodge we have sought to elucidate the source of electromagnetic action between a primary circuit carrying alternating current and a secondary circuit in which an alternating voltage is induced. Without any form of electromagnetic shielding present the induced voltage was shown to be consistent with standard mutual inductance calculations based on $e=M d I / d t$. With various forms of shielding present around the circuits (so reducing the static magnetic field in the vicinity of the secondary to nearly zero) the magnitude of the induced voltage remained unchanged within the limits of experimental error. Now in the case of an infinite solenoid the electric field has an explanation in terms of vector potential. The vector potential outside the solenoid is not zero and varies with time so creating an electric field (Equation 2).

So are we still entitled to ask has the vector potential A any physical significance? According to Maxwell's point of view it is a condition for motion of stress in a medium already existing in space (aether). Consequently, by acceptance of vector potential as a physical entity we seem to return to the aether of the $19^{\text {th }}$ century that is, in fact, the aether of Maxwell, Lorentz and Lodge, but now seen from a somewhat different perspective. The discovery of the Aharonov-Bohm effect in 1959 seemed to provide further evidence for the significance of vector potential. The shift in the fringe pattern between two electron beams passing across and either side of a long current carrying solenoid should not exist if it is assumed that only the magnetic field can affect the electrons. Nevertheless a mathematical treatment involving both vector potential and the Schrodinger equation demonstrated that a quantum force is present even when the magnetic field is zero.

Clearly the $\mathbf{E}$ and $\mathbf{B}$ fields of the traditional Lorentz force law equation are not sufficient to explain either the A-B effect or the case of transformer induction considered in the present paper. However the question is posed as to whether classical physics can be adapted to provide explanations without invoking quantum mechanics? The Lorentz force law may be well in need of modification. It has been criticised in that it attempts to combine two incompatible cases -- that of a force on a static charge in combination with a force on a moving charge in a magnetic field. Another approach might be through Weber's Electrodynamics based on action at a distance. Such theories are pre-Maxwellian and involve only forces between moving charges; they do not involve directly $\mathbf{E}$ and $\mathbf{B}$ vectors as 'field' quantities. In his 1889 paper Lodge refers, rather disparagingly, to the likes of Weber's work as 'contemporary German theories'. As a fervent believer in the aether, Lodge would have instinctively preferred Maxwell to Weber! Our suggestion is to renounce the prevailing "kinematical" point of view based on the admission of 'magnetic field lines' movement, and to substitute it for the pure dynamical model, free of any reference to the Lorentz force. So given that a variable electric field is present in a location of negligibly small magneto-static field while by contrast the vector potential has a distinct value, we would conclude that vector potential rather than magnetic field provides a causal explanation of the induced voltage in the experiments outlined.

The questions discussed in this paper certainly present an interesting subject for further study. In the modern teaching of electromagnetism, as exemplified in our current textbooks, there is a long-standing order of settled ideas and stable opinions. However, physics is a natural science, and always experiment should be the final arbiter between competing theories. Through the results obtained in this present investigation, we hope to revive the scientific community's interest and curiosity in this aspect of classical electrodynamics.

\section{Acknowledgements}

We sincerely thank Mr. Alan Edwards, at the University of Liverpool, for providing the adjustable power amplifier used for our experimental work.

\section{References}

Aharonov, Y., \& Bohm, D. (1959). Phys. Rev., 115, 485.

Bogach, V. A. (1996). A hypothesis about the existence of a static electromagnetic field and its properties (Preprint 13-96-463). Dubna, Russia: JINR (in Russian).

Feynman, R. P., Leighton, R. B., \& Sands, M., (1989). The Feynman Lecture on Physics (Vol. 2). Reading, Massachusetts: Addison-Wesley.

Grant, I. S., \& Phillips, W. R. (1998). Electromagnetism (2nd ed.). Chichester: John Wiley \& Sons.

Gray, A. (1967). Absolute Measurement in Electricity and Magnetism. New York, NY: Dover Publications.

Kelly, A. G. (2004). Unipolar experiments. Annales de la Foundation Luis de Broglie, 29(1-2), 119-148.

Leus, V. A., \& Taylor, S. (2011). On the motion of the field of a permanent magnet. Eur. J. Phys., 32(5), 1179-1192. http://dx.doi.org/10.1088/0143-0807/32/5/006

Leus, V. A., \& Taylor, S. (2012). Experimental Evidence for the Magneto-kinematic Effect. PIERS Proceedings, 
Moscow, Russia, August 19-23, 1040-1048.

Leus, V. A., \& Zatolokin, V. N. (2006). The magnetokinematical effect. IJEEE, 43(4), 245-251.

Lodge, O. (1889). On an Electrostatic Field produced by varying Magnetic Inducion. Phil. Mag., 27, 469-479.

Mould, R. A. (1995). Basic Relativity. New-York: Springer-Verlag.

O’Rahilly, A. (1965) Electromagnetic Theory (Vol. 1). New York: Dover Publications.

Poynting, J. H. (1885). On the Connexion between Electric Current and the Electric and Magnetic Inductions in the Surrounding Field. Philosophical Transactions of the Royal Society, 176, 277-306. http://dx.doi.org/10.1098/rstl.1885.0003

Rajaraman, K. C. (2008). The field of a rotating cylindrical magnet. IJEEE, 45(1), 34-45.

Rousseaux, G., Kofman, R., \& Minazzolli, O. (2008) The Maxwell-Lodge effect: significance ofelectromagnetic potentials in the classical theory. The European Physical Journal D, 49, 249-256. http://dx.doi.org/10.1140/epjd/e2008-00142-y.

Taylor, S., \& Leus, V. A. (2012). The magneto-kinematic effect for the case of rectilinear motion. Eur. J. Phys., 33(4), 837-852. http://dx.doi.org/10.1088/0143-0807/33/4/837.

Tonomura, A. (1998). The quantum world unveiled by electron waves. World Scientific.

Zajev, N. E., \& Dokuchajev, V. I. (1964). About behaviour of force lines of the field of a magnet. Electrotechnika (Electrical engineering, in Russian), 11, 64.

\section{Copyrights}

Copyright for this article is retained by the author(s), with first publication rights granted to the journal.

This is an open-access article distributed under the terms and conditions of the Creative Commons Attribution license (http://creativecommons.org/licenses/by/3.0/). 